\title{
Наноэлектроэрозия: коррекция влияния среды зазора «swcnt-электрод-поверхность»
}

\author{
С. М. Слободян \\ Омский государственный технический университет, \\ 2. Омск, 644050, Россия, e-mail: sms 46@ngs.ru \\ Поступила в редакцию 01.09.2020 \\ После доработки 21.10.2020 \\ Принята к публикации 21.10.2020
}

\begin{abstract}
Рассмотрена возможность коррекции проявления искажающего действия случайного слоя среды, окружающей и заполняющей промежуток «swcnt-электрод-поверхность» в системах электроэрозионной обработки материалов, проводящих электрический ток. Сущность восстановления сигнала основана на процедуре коррекции искажения реального сигнала электроэрозионного воздействия и базовых положениях теории цифровой фильтрации. Малое значение среднеквадратической погрешности коррекции при восстановлении сигнала электроэрозионного воздействия, полученной моделированием, указывает на улучшение качества сигнала электроэрозионного воздействия.
\end{abstract}

Ключевые слова: искажение, коррекция, случайная среда, нанозазор, «swcnt-поверхность»

УДК 621.9.048:621.89:621.37

https://doi.org/10.52577/eom.2021.57.2.34

ВВЕДЕНИЕ

Среда промежутка «swcnt-электрод-поверхность», которая разделяет поверхность электрода, выполненного из однослойной углеродной трубки (англ.: swcnt-simple-walled carbon nanotube), и поверхность обрабатываемого изделия представляет в данной работе объект исследования.

В простом геометрическом представлении [1-6] пространство промежутка «анод-катод» активной электроэрозионной зоны воздействия на материал ограничено двумя поверхностями: поверхностью активного электрода - swcnt (анод) и поверхностью обрабатываемого материала, служащего катодом - источником эмиссии (от электронов до отдельных капель абляции структуры) элементов материала. В пространстве промежутка активной электроэрозионной зоны действия присутствует среда, которая разделяет два электрода $[1,3,6,7-10]$ : один - swcnt (анод) является инструментом, второй (катод) - материалом интенсивного электротермического воздействия. Из-за того, что в процессе формирования активной электроэрозионной зоны действия происходит совершенно случайный отрыв фрагментов структуры материала от поверхности обрабатываемого изделия, геометрия промежутка «swcntповерхность» динамически изменяется. Структура и состав слоя среды, заполняющий промежуток «swcnt-поверхность» активной электроэрозионной зоны действия, так же как и пространство промежутка «swcnt-поверхность» являются случайно меняющимися во времени параметрами. Это обусловлено, в первую очередь, значительным пространственным и временным изменениями, в большей степени из-за абляции фрагментов, поверхности обрабатываемого материала. Кроме того, что вполне естественно, эмитировавшие в пространство промежутка «swcnt-поверхность» фрагменты материала изменяют состав и структуру среды промежутка. Таким образом, состав и структура слоя среды в промежутке активной электроэрозионной зоны действия между электродами «swcnt-электрод-поверхность» подвержены сильным случайным пространственным и временным изменениям в динамике протекания процесса [1, 3, 6, 9-14]. Наиболее неустойчивым распределением составляющих отличается слой в центральной части зазора, особенно его пограничные слои, которые прилегают к поверхности swcnt и поверхности изделия. Именно здесь происходит формирование «абляционного» характера процесса разрушения изотермических слоев среды и аэрозолей материала [1, 10-15].

Интенсивная динамика перемешивания компонентов (газовой, жидкостной и частиц) слоя среды в зазоре и вымывание конвективными или принудительными потоками ее движения обусловливают кратковременность пребывания в этом слое ее компонентов, сформировавших структуру слоя среды на одном из циклов наблюдения в каждом из элементов слоя пространства зазора. В общем случае она 
представляет собой смесь основных фоновых составляющих окружающей промежуток среды (жидкостной или газовой) и наиболее типичных концентраций частиц материала обработки как включений основной среды. Термически и термодинамически активные компоненты основных концентраций частиц материала обработки оказывают наиболее сильное влияние на свойства среды промежутка даже при ничтожно малых их концентрациях в слое среды «swcntповерхность».

C развитием наноразмерных технологий наблюдается высокий интерес к решению проблем электроэрозионного сверления отверстий малого диаметра $[6,8,9,15-31]$. Например, для области наноэлектроники необходимый диаметр отверстий соизмерим с долями нанометра [9, 15-18]. Создание отверстий-ниш в наноразмерных проводниках и размещение в таких нишах отдельных элементов цепей, несомненно, повышают плотность интеграции схем. Качество получаемых при этом отверстий зависит от степени засорения среды промежутка «электрод-поверхность» продуктами эрозии из зоны обработки. Поэтому решение проблемы снижения влияния на сигнал электроэрозионного воздействия степени засорения продуктами эрозии среды промежутка весьма важно для повышения качества сверления.

Засорение продуктами эрозии среды промежутка «электрод-поверхность» из-за их неэффективной эвакуации из зоны обработки ослабляет сигнал электроэрозионного воздействия на материал, ухудшает точность формообразования и ограничивает глубину формируемых отверстий, снижая высокую производительность прошивки отверстий. Поэтому решение задачи восстановления исходно требуемого сигнала электроэрозионного воздействия на материал, например, путем компенсации искажений сигнала воздействия является важной необходимостью, как и создание методов более эффективного удаления из зазора «электродповерхность» частиц эрозионного разрушения и газового пузыря - сильного препятствия для формирования разряда.

В плане совершенствования существующих методов и средств повышения производительности электроэрозионного воздействия, являющихся главным показателем эффективности технологии и остающихся одним из основных условий сохранения и повышения ее конкурентоспособности, рассмотрим возможность применения коррекции искажающего действия случайного слоя среды, которая заполняет промежуток «электрод-поверхность» в системе электроэрозионного действия.
Наличие указанных проблем определяет интерес к анализу и оценке возможностей способов коррекции искажений сигнала электроэрозионного воздействия и управления для улучшения качества и точности формообразования при прошивке отверстий.

Цель работь - исследование возможности создания, основанного на положениях теории фильтрации и данных измерения в реальном времени, метода компенсации искажающего воздействия среды, динамически заполняющей случайно изменяющийся промежуток «swcntэлектрод-поверхность» в системе электроэрозионного действия.

\section{МЕТОДЫ ИССЛЕДОВАНИЯ}

Методы исследования используемые в данной статье основаны на положениях физики, теорий случайных процессов, фильтрации и обработки электроэрозионного сигнала воздействия $\mathrm{c}$ учетом неэффективной эвакуации продуктов эрозии из зоны обработки материала. В исходной постановке мы учитываем фактор наличия продуктов эрозии в среде промежутка «электродповерхность» и диэлектрического газового пузыря - неизменного спутника электроэрозионной технологии в любой охлаждающей среде промежутка «электрод-поверхность». Потому в данной работе, чтобы упростить оценку исследуемых возможностей предлагаемого метода рассматриваем процесс электроэрозионного сигнала воздействия с охлаждением промежутка «электрод-поверхность» зоны обработки материала воздушно-газовой средой. Воздушно-газовая среда, окружающая зону обработки, обладая высокими диэлектрическими свойствами является сильным препятствием для формирования разряда - главного элемента инструмента эрозионного разрушения.

Искажающее действие случайной структуры и состава среды, которая динамически заполняет промежуток «электрод-поверхность», в цепи формирования сигнала электроэрозионного дугового воздействия на материал, в принимаемой линейной модели системы электроэрозионной обработки можно описывать математической операцией свертки исходно формируемого, неискаженного сигнала электрического воздействия $\mathrm{c}$ импульсной характеристикой среды промежутка «электрод-поверхность», имеющей вид:

$$
\begin{aligned}
& f(x, y)=\varphi(x, y) \square h(x, y)= \\
& =\iint \varphi(x, y) h(x-\eta, y-v) d \eta d v,
\end{aligned}
$$

где $f(x, y)$ - искаженный средой промежутка «электрод-поверхность» сигнала электрического 
воздействия; $\varphi(x, y)$ - исходно формируемый, неискаженный электрический сигнал; $h(x, y)-$ импульсная характеристика среды, искажающей сигнал в промежутке «электрод-поверхность» элемента цепи формирования сигнала электроэрозионного дугового воздействия; - знак операции «свертка»; $\iint$ - интегрирование уравнения (1) осуществляется в пределах $[-\infty ;+\infty] ; \eta$ и $v$ - параметры интегрирования.

Формально составляющую электрического шума, естественно присутствующую аддитивно в цепи формирования сигнала электроэрозионного дугового воздействия на промежуток и материал, мы, в данном исследовании, не учитываем по двум причина. Во-первых, она легко устраняется специальными методами, зависящими от конкретного типа источника шумового воздействия [10-12, 14-17]. Во-вторых, опускаем ввиду несущественности действия фактора на результат анализа, что позволяет упростить математическое описание алгоритма коррекции влияния среды промежутка «электродповерхность» на сигнал электроэрозионного дугового воздействия.

В отношении электромагнитных волн и сигналов импульсную характеристику элемента цепи передачи электромагнитного сигнала электроэрозионного дугового воздействия [15-19] можно считать функцией рассеяния некоторой пространственно ограниченной области, в пределе, - точки. Для промежутка «электрод-поверхность», заполненного некоторой средой, функция рассеяния точки может быть определена из совокупности проведения теоретических расчетов и статистических моделей, основанных на данных экспериментальных исследований и практики производственных работ, например путем прямых измерений, проводимых в реальном процессе формирования сигнала электроэрозионного дугового воздействия на материал [14, 18-31].

В динамике движения электрода с учетом наличия неравномерностей рельефа поверхности в промежутке «электрод-поверхность», размер указанного промежутка не остается постоянным, как принято считать. Размер промежутка «электрод-поверхность», следовательно, и толщина слоя среды с включениями частиц материала, которая его заполняет, меняются случайным образом в соответствии с изменением рельефа обрабатываемой поверхности. Такое случайное изменение свойств рассеивающего электромагнитные волны слоя среды, заполняющей промежуток «электрод-поверхность», в реальном времени вносит случайные искажения в форму электромагнитного сигнала электроэрозионного дугового воздействия на материал.
Эти динамически случайные искажения в объеме промежутка не являются постоянными. Они вероятно распределены из-за неоднородности структуры слоя среды промежутка «электрод-поверхность».

Изложение статьи проведем на примере рассмотрения процесса наноэлектроэрозии с «swcnt-электродом», являющимся однослойной нанотрубкой (single-walled carbon nanotube swcnt), представляющей собой одноатомный лист графена, свернутый в форму цилиндра [16-27]. Свертка одноатомного листа графена в форму цилиндра в сильной степени, скажем даже, - в мере определяющей результат процесса свертки, зависит от хиральности, свойств и особенностей топологии распределения атомов по площуади одноатомного листа [20-21]. Bымеуказанные особенности свертки листа графена в циллинр приводят к формированию swcnt c тремя типами свойств: металлические, полупроводниковые и прочие swcnt.

Важно отметить, что первыми исследователями, апробировавшими возможность применения swcnt-электрода как инструмента для электроэрозионного дугового воздействия на материал, были авторы работы [18]. Опубликованные ими результаты экспериментов, показали, что swcnt-электрод как инструмент для электроэрозионного дугового воздействия, благодаря малому диаметру способен проникать в отверстия на поверхности исследуемого материала диаметром 0,4 мкм и глубиной 0,8 мкм. До сих пор этот эксперимент является уникальным для многих практик. Кроме того, приложив к swcnt-электроду как электроэрозионному инструменту импульс напряжения, они нанесли на дно вымеуказанного отверстия пятнышко углерода диаметром 40 нм с визуализачией его изображения. Указанные факты говорят о том, что электропроводящче свойства нанотрубок, не только углеродных и однослойных, но и многослойных, позволяют организовать их иирокое применение как основы наноэлектрода в электроэрозионном инструменте.

Учитывая предельно малые размеры swcntэлектрода, функцию рассеяния тока в пространстве промежутка «swcnt-электродповерхность», заполненного окружающей средой с включениями частиц разрушения материала, вносящую искажения, можно принять симметричной относительно центра swcnt-электрода электроэрозионного инструмента. В общем случае, функция рассеяния тока, протекающего через swcnt-электрод, будет сосредоточена на четырех смежных квадрантах элемента, противолежащего swcnt-электроду с центром, совпа- 
дающим с центром цилиндра swcnt-электрода в плоскости $X Y$ системы координат. Из-за неоднородности структуры среды и поперечного сечения swcnt-электрода, функция рассеяния тока в слое промежутка «swcnt-электродповерхность» будет несимметрична. Теоретически функции рассеяния тока в слое среды свойственна бесконечная протяженность в пространстве.

Восстановление искаженного сигнала электроэрозионного дугового воздействия на материал с учетом принятого нами предположения об отсутствии шума сводится к нахождению импульсной характеристики системы восстановления сигнала, являющейся обратной по отношению к импульсной характеристике системы, вызвавшей его искажения. Условие точного восстановления сигнала электроэрозионного воздействия соответствует выполнению следующего равенства:

$$
h(x, y) \square h^{\prime}(x, y)=\delta(x, y),
$$

где $h^{\prime}(x, y)$ - импульсная характеристика системы восстановления сигнала электроэрозионного воздействия; $\delta(x, y)=1(x, y)-$ единичная функция. На практике, из-за влияния многих факторов, идеальное получение равенства $\delta(x, y)=1(x, y)$ затруднено. Гораздо чаще, получаем $\delta^{*}(x, y) \approx 1$, где $\delta^{*}(x, y)$ - некоторое приближение результата к идеальному решению $\delta(x, y)=\delta^{*}(x, y)$.

В операторном представлении уравнение (1) искажающего действия слоя среды, динамически заполняющей промежуток «swcnt-электродповерхность», в линейной модели цепи формирования $f$ сигнала электроэрозионного воздействия примет вид:

$$
f=H \varphi,
$$

где $H$ - линейный интегральный оператор цепи формирования сигнала электроэрозионного воздействия; $\varphi$ - исходный сигнал электроэрозионного воздействия.

Если предположить, что существует оператор $B$, обратный по отношению к линейному оператору $H$, такой, что

$$
B=H^{-1}
$$

тогда, следуя (4), получим операторное уравнение (3) следующего вида:

$$
B f=\varphi \text {. }
$$

Операторное уравнение (5) следует понимать как уравнение относительно неизвестной функции $f$ при известных функции $\varphi$ и параметрах оператора восстановления сигнала эрозионного воздействия $B$, обратного по отношению к линейному оператору искажений сигнала $H$. При заданных начальных условиях $f_{\mid(0,0)}=f_{0}$ операторное уравнение (5) первого порядка должно иметь единственное решение. Оператор восстановления сигнала $B$ своими параметрами полностью характеризует линейную систему формирования неискаженного сигнала воздействия из искаженного в цепи промежутка «swcnt-электрод-поверхность» [10, 15-17, 19, 30-32].

Контроль ширины зазора, часто проводят путем измерения падения напряжения на зазоре методом емкостной связи [10-11, 13-19, 22-24, 26-31]. Физические явления, которые формируют процесс электроэрозионного воздействия на материал, в общем случае достаточно сложны и многогранны. Процесс электроэрозионного воздействия на материал является многоэтапным. Физической основой разрушительного действия процесса электроэрозионного воздействия на структуру обрабатываемого материала является электрический разряд между «swcnt-электродом» и поверхностью. На начальном этапе происходит электрический пробой. Электрический пробой сопровождается искровыми разрядами. Затем, по мере развития процесса ионизации, из структуры искровых разрядов формируется установившийся дуговой разряд. Наличие многоэтапного протекания многогранной структуры физических явлений электроэрозионного воздействия дает возможность проведения контроля параметров электроэрозионного процесса с привлечением широкого спектра - оптических, акустических и других методов диагностики и визуализации процесса электроэрозионного воздействия, в том числе на технологической стадии [10-19, 27-31]. При этом следует учитывать влияние на динамику процесса электроэрозии множества эффектов, в том числе, резонансного взаимодействия «swcnt-электрод-поверхность», вызванных электрическим разрядом в цепи промежутка «swcnt-электрод-поверхность» [29-31].

\section{РЕЗУЛЬТАТЫ}

Таким образом, приведенные выше операторные уравнения (3)-(5) алгоритма восстановления сигнала электроэрозионного воздействия, искаженного средой с включениями частиц материала в промежутке «swcnt-электродповерхность», позволяют по известному входу и выходу линейной системы формирования сигнала эрозионного воздействия определять параметры искажающего воздействия и восстанавливать сигналы электроэрозионного воздей- 
ствия, характеризующиеся разной степенью искажений относительно заданного эталона. Действие алгоритма восстановления сигнала воздействия, описываемого уравнениями (3)-(5), было апробировано нами на практике при решении задачи коррекции линейной моды фазовых искажений волнового фронта лазерного пучка в активной системе с обратной связью [32].

Коррекция искажающего действия слоя среды с включениями частиц материала, динамически заполняющей промежуток «swcnt-электродповерхность», даже в линейной цепи формирования сигнала электроэрозионного воздействия имеет существенные отличия. Часть отличий приведена ранее. В частности, случайность и динамичность изменения слоя среды с включениями частиц материала ведет к тому, что условие (2) точного восстановления сигнала электроэрозионного воздействия становится трудновыполнимым.

В отсутствие эталона условие (2) восстановления сигнала электроэрозионного воздействия превращается в неравенство:

$$
h(x, y) \square h^{\prime}(x, y)=\delta(x, y)<1(x, y) \text {. }
$$

В таком случае при заданной импульсной характеристике искажающей среды как фильтра, определение коэффициентов фильтра $\gamma_{r s}^{\prime}$ находится решением уравнения идентификации вида:

$$
\operatorname{Bh}(x, y)=\delta(x, y) \text {, }
$$

где $B$ - оператор линейной системы, параметры $h(x, y)$ и $\delta(x, y)$ - имеют прежний смысл, указанный в (2) и (4).

В дискретном представлении $h_{i j}$ и $\delta_{i j}$ определяются в равномерном квадратном растре разбиения элемента воздействия $i=-M . . .0 . . M$, $j=-M \ldots 0 . . . M, \delta_{i j}=[1, i=j=0 ; 0$, в остальных случаях, когда $i \neq j \neq 0, i=j \neq 0$ и т.д.]. При заданном начальном условии $h\left(x_{0}, y_{0}\right)=h_{0}$ уравнение (6) имеет единственное решение.

Применив к дискретному уравнению двумерное $z$-преобразование, получим

$$
B\left(z_{1}, z_{2}\right) H\left(z_{1}, z_{2}\right)=1 \text {. }
$$

Отсюда находим передаточную характеристику искажающего воздействия среды промежутка «электрод-поверхность»:

$$
H\left(z_{1}, z_{2}\right)=1 / B\left(z_{1}, z_{2}\right) .
$$

Если повторить выше изложенные преобразования применительно к уравнению (7), то получим выражение импульсной характеристики искажающей среды промежутка «электродповерхность» вида рекурсивного фильтра:

$$
h_{i j}=\delta_{i j}-\sum \gamma_{r s} h_{i-r, j-s}, \text { для }(r, s) \neq(0,0) \text {; }
$$

а условие восстановления сигнала воздействия вида описания нерекурсивного фильтра

$$
\delta_{i j}=\sum \gamma_{r s} h_{i-r, j-s} \text {. }
$$

Для определения коэффициентов $\gamma_{r s}^{\prime}$ следует минимизировать сумму

$$
\sum\left[\delta_{i j}-\sum \gamma_{r s}^{\prime} h_{i-r, j-s}\right]^{2} \rightarrow \min \left(\gamma_{r s}^{\prime}\right)
$$

Применяя метод наименьших квадратов, получим систему линейных уравнений, из решения которой найдем требуемые значения коэффициентов фильтра $\gamma_{r s}^{\prime}$ коррекции искажений сигнала воздействия.

Для минимизации числового значения погрешности решения уравнения (6), импульсная характеристика искажающего слоя среды $h_{i, j}$ промежутка «Swcnt-электрод-поверхность» должна содержать в (10) как можно большее число дискретных отсчетов. В идеале их число должно быть бесконечным. На практике для решения проблемы коррекции сигнала электроэрозионного воздействия достаточно примерно десятой доли несоответствия импульсных характеристик.

Выбор порядка $p$ оператора восстановления сигнала электроэрозионного воздействия основан на следующем. Во-первых, желателен учет априорной информации о характере искажающего влияния среды промежутка »swcntэлектрод-поверхность». Например, импульсную характеристику рассеивающей ток в среде промежутка «swcnt-электрод-поверхность» как элемента цепи формирования сигнала электроэрозионного воздействия можно представить [15] экспонентой с показателем первой степени $h \sim \exp (-x)$. Этому соответствует линейный оператор в уравнении (6). Во-вторых, если нужно повысить точность решения уравнения (6), следует итерационным методом выполнить увеличение порядка уравнения линейного оператора.

Подводя итог проделанной работе, отметим следующее.

Природный процесс искажения сигнала электроэрозионного воздействия, в виде вносимого средой промежутка «swcnt-электродповерхность» запаздывания формирования и коррекции сигнала электроэрозионного воздействия, характеризуется откликом бесконечной длины. Мы описываем его действием физически нереализуемого рекурсивного фильтра, также с бесконечной импульсной характеристикой. При этом синхронность процессов искажения и коррекции сигнала электроэрозионного воздей- 
Таблица. Среднеквадратическая ошибка коррекции

\begin{tabular}{|c|c|c|c|c|c|c|c|c|}
\hline $\mathrm{M}$ & 1 & 2 & 3 & 4 & 5 & 6 & 7 & 8 \\
\hline$\varepsilon(p=0)$ & 0,33 & 0,20 & 0,14 & 0,11 & 0,09 & 0,08 & 0,07 & 0,06 \\
\hline$\varepsilon(p=1)$ & 1 & 0,33 & 0,20 & 0,14 & 0,11 & 0,09 & 0,08 & 0,07 \\
\hline$\varepsilon(p=2)$ & -1 & 1 & 0,33 & 0,20 & 0,14 & 0,11 & 0,09 & 0,08 \\
\hline
\end{tabular}

ствия с достаточной степенью точности описывает выражение (8).

Второй важный момент: на процесс динамического искажения сигнала электроэрозионного воздействия средой промежутка «swcntэлектрод-поверхность» как элемента цепи его формирования повлиять мы не можем. В системах электроэрозионной обработки он составляет сущность принципа процесса электроэрозионного воздействия. С точки зрения устойчивости рекурсивной фильтрации можно предположить, что устойчивому процессу коррекции искажений сигнала электроэрозионного воздействия соответствует устойчивый рекурсивный фильтр.

Опуская несложные преобразования, точность коррекции искаженного средой промежутка «swcnt-электрод-поверхность» сигнала электроэрозионного воздействия можно количественно оценить значением $\varepsilon-$ среднеквадратического отклонения значения разности двух процессов, квадрат которого является $\varepsilon^{2}-$ среднеквадратической ошибкой коррекции, равной

$$
\varepsilon^{2}=\left[\sum\left(\delta_{i j}^{\prime}-\delta_{i j}\right)^{2}\right] /(2 M+1-2 p)^{2},
$$

где $\delta_{i j}^{\prime}$ - оценка импульсной переходной характеристики рассеивающего слоя среды промежутка «swcnt-электрод-поверхность» - элемента цепи формирования сигнала электроэрозионного воздействия; $\delta_{i j}$ - импульсная характеристика искажающей среды промежутка «swcntэлектрод-поверхность» как рекурсивного фильтра; $\Sigma$ - знак операции суммирования невязки $\left(\delta_{i j}^{\prime}-\delta_{i j}\right)$ по всем $i j ; M-$ число элементов растра разбиения области электроэрозионного воздействия; $p$ - порядок уравнения оператора восстановления. Изменение величины численной оценки точности коррекции сигнала электроэрозионного воздействия, искаженного действием среды промежутка «swcnt-электрод-поверхность» - элемента цепи формирования сигнала электроэрозионного воздействия в виде числового значения (11) среднеквадратического отклонения, нормированного на $\Sigma\left(\delta_{i j}^{\prime}-\delta_{i j}\right)$, приведено в таблице для трех (нулевого, первого и второго) значений $p$ - порядка уравнения оператора восстановления.

Знак минус в значении среднеквадратического отклонения $\varepsilon$ в таблице при использовании уравнения второго порядка для оператора восстановления означает перекомпенсацию величины искажений сигнала воздействия. Учитывая результаты работ [1, 15, 18-19] и фундаментальные положения теории подобия, можно говорить об улучшении качества электроэрозионного воздействия.

Отметим важные особенности действия коррекции сигнала электроэрозионного воздействия, искаженного средой промежутка «swcnt-электрод-поверхность».

Во-первых, следует уточнить: предложенная методика коррекции сигнала электроэрозионного воздействия, в основном, учитывает действие на сигнал диэлектрической среды газа в промежутке «swcnt-электрод-поверхность» при незначительной глубине отверстий, когда сохраняется параллельность боковых поверхностей формируемого отверстия и swcnt-электрода и без учета ионизации газовой среды промежутка. Таким образом, границы применимости предложенной методики на данной стадии исследования ограничены условиями газовой среды промежутка.

Во-вторых, синхронное движение слоя диэлектрической среды с проводящими включениями частиц материала в промежутке «swcntэлектрод-поверхность» [22-25] может достаточно сильно влиять на эффективность коррекции сигнала электроэрозионного воздействия. Динамика движения среды и частиц в изложенном методе коррекции искажений сигнала электроэрозионного воздействия средой промежутка «swcnt-электрод-поверхность» не принималась во внимание. Ее учет и аспекты резонансного характера влияния в силу вероятностной природы [22, 29-32] необходимы для повышения точности оценки коррекции сигнала электроэрозионного воздействия, если это является необходимостью.

\section{ЗАКЛЮЧЕНИЕ}

Исследована возможность коррекции сигнала электроэрозионного воздействия искаженного действием слоя окружающей среды, заполняющей промежуток «swcnt-электродповерхность» при электроэрозионной обработке материала, проводящего ток. Основой коррекции служит сигнал электроэрозионного воздействия в системе обработки. Учтено искажающее действие среды в промежутке «swcnt-электродповерхность», имеющее вероятностную природу. 
Выполнена оценка эффективности коррекции искажающего действия промежутка «swcntэлектрод-поверхность». Результаты работы применимы в практике систем электроэрозионной обработки метаматериалов и наноструктур. Показано, что применение нанотрубок является технически оправданным.

\section{БЛАГОДАРНОСТЬ}

Выражаю искреннюю благодарность всем коллегам, в разной мере повлиявшим на результат выполнения данной работы.

\section{ЛИТЕРАТУРА}

1. Боков, В.М., Сиса, О.Ф., Использование электрической дуги для получения металлических порошков, ЭОМ, 2019, т. 55, № 2, с. 60.

2. Боков, В.М., Сиса, О.Ф., Мирзак, В.Я., Механизм формообразования плоских поверхностей электрической дугой, ЭОМ, 2018, т. 54, № 3, с. 53.

3. Deeva, V., Slobodyan, S., Effect of pressure changes in sliding contact, Int. J. Eng. Technol. 2018, vol. 7, no. 2, p. 167.

4. German, R.M., Progress in titanium metal powder injection molding, Materials, 2013, vol. 6, no. 8, p. 3641.

5. Angelo, P.C., Micro and nano fabrication by powder metallurgy. J. Powder Metall. Min., 2015, vol. 4, no. 1 , p. 1.

6. Малюшевская, А.П., Малюшевский, П.П., Оптимизация процесса тонкого электроразрядного диспергирования, ЭОМ, 2019, т. 55, № 3, с. 51.

7. Deeva, V., Slobodyan, S., Martikyan, M., Physical model of the sliding contact of conductors of the alloy $\mathrm{Cu}-\mathrm{Zr}$ and $\mathrm{Cu}-\mathrm{Re}$ under high current density, Mater. Today Proceed., 2016, vol. 3, no. 9ptB, p. 3114.

8. Deng, Z.Q., Zhu, Y.W., Wang, F., Gu, X., et al., Analysis and experimental study of vibration system characteristics of ultrasonic compound electrical machining, Strength. Mater., 2017, vol. 49, p. 37.

9. Груздев, А.А., Моргунов, Ю.А., Саушкин, Б.П., Электроэрозионная прошивка отверстий малого диаметра при наложении ультразвука. Часть 1. Результаты технологических экспериментов, ЭОМ, 2019, т. 55, № 1, с. 12.

10. Furusato, T., Yamamoto, Y., Sakamoto, T., Oura, K., et al., Simulation of voltage/current waveforms and contact area of pulsed surface discharge on water. IEEE Trans. Dielectr. Electr. Insul., 2019, vol. 26, no. 2, p. 439.

11. Bol'shanin, A.A., Slobodyan, S.M., Yakovlev, A.R., Vasil'eva, L.A., Two-channel optical transducer for an industrial inspection system, Meas. Tech., 1987, vol. 30, no. 10, p. 954.

12. Slobodyan, M.S., Shishigin, S.A., Slobodyan, S.M., Method of acoustic sensor diagnosis, Meas. Tech., 2008, vol. 51, no. 7, 798 .
13. Graphene: Fabrication, Characterizations, Properties and Applications. Editors by: H. Zhu, Z. Xu, D. Xie, Y. Fang. Beijing: Academic Press, 2018.

14. Arutyunov, V., Mel'nikov, V., Slobodyan, S., Chaporov, D., et al. Sources of measurement error of fast-flowing process parameters by charge-coupled devices, Meas. Tech., 1983, vol. 26, no. 8, p. 641.

15. Burke, P.J., An RF circuit model for carbon nanotubes, IEEE Trans. Nanotechnol., 2003, vol. 2, p. 55.

16. Volkov, V., Peshel', A., Slobodyan, S., Tyryshkin, I.S., Registration of a pulsed laser beam by a matrix of charge-coupled devices, Instrum. Exp. Tech., 1981, vol. 24, p. 1522.

17. Gonzalez, R., Woods, R., Digital Image Processing, New-York: Pearson, 2018. 1192 p.

18. Dai, H., Hafner, J., Rinzler, A., Colbert, D., et al., Nanotubes as nanoprobes in scanning probe microscopy, Nature, 1996, vol. 384, p. 147.

19. Deeva, V., Slobodyan, S., Mathematical model of tip oscillations: influence on image quality, Appl. Surf. Sci., 2020, vol. 516, 146144.

20. Geim, A.K., Novoselov, K.S., The rise of grapheme, Nat. Mater., 2007, vol. 6, p. 183.

21. Silva J., Lanceros-Mendeza S., Simoes R. Effect of cylindrical filler aggregation on the electrical conductivity of composites. Phys. Lett. A., 2014, vol. 378, p. 2985.

22. Deeva, V.S., Slobodyan, S.M., Teterin, V.S., Optimization of oil particles separation disperser parameters, Mater. Sci. Forum. 2016, vol. 870, p. 677.

23. Tang, C., Long, G., Hu, X., Wong, K., et al., Conductive polymer nanocomposites with hierarchical multiscale structures via self-assembly of carbon nanotubes on graphene on polymer-microspheres, Nanoscale. 2014, vol. 6, p. 7877.

24. Ryabtseva, N., Barchukov, D., Slobodyan, S., Karelin, A., Correlation method of estimation of the relief impact synchronicity on the CNT-needle and probe. 2020 IEEE 40th International Conference on Electronics and Nanotechnology (ELNANO), Kyiv, Ukraine, 2020, pp. 18-21, doi: 10.1109/ELNANO50318.2020.9088814.

25. Kim, S.H., Haines, C.S., Li, N., Kim, K.J., et al., Harvesting electrical energy from carbon nanotube yarn twist, Science, 2017, vol. 357, p. 6353.

26. Li, Y., Single-atom catalysts: The role of intrinsic intermediate, Green Energy Environ., 2020, vol. 5, p. 4.

27. Nie, M., Kalyon, D.M., Fisher, F.T., Interfacial load transfer in polymer/carbon nanotube nanocomposites with a nanohybrid shish kebab modification, ACS Appl. Mater. Interfaces. 2014, vol. 6, no. 17, p. 14886.

28. Deeva, V., Slobodyan, S., Assessment of the tribological contact between sliding surfaces via an entropy approach. J. Tribol-T. ASME. 2019, vol. 141, no. 3, 031602. 
29. Kosenkov, V.M., Resonant vibrations of a cylindrical wall of a discharge chamber, induced by an electrical discharge in water, J. Appl. Mech. Tech. Phys., 2011, vol. 52, no. 4, p. 536.

30. Romanishina, S.A., Katyuk, D.Y., Deeva, V.S., Slobodyan, S.M., Dynamics layer of the sliding contact collector elements, 2015 IEEE 35th International Conference on Electronics and Nanotechnology (ELNANO), Kiev, 2015, pp. 116-118. doi: 10.1109/ELNANO.2015.7146848.

31. Seriani, S., Medvet, E., Carrato, S., Gallina, P., A complete framework for the synthesis of powered floor systems, IEEE/ASME Trans. Mechatron., 2020, vol. 25, no. 2, p. 1045.
32. Slobodyan, M.S., Slobodyan, S.M., Tsupin, A.A., Optical deflector of a wave-front tilt corrector, J. Opt. Technol., 2008, vol. 75, no. 5, p. 301.

\section{Summary}

A possibility of correction of the distorting effect of a random environment layer that fills a nanoscale "swcnt-electrode-surface" gap in systems of electroerosive processing of conducting current materials is considered. Correction of the nano-erosion impact signal is based on the principles of the digital filtering theory. A small value of the root-mean-square error of distorted signal correction of an electroerosive effect, obtained by modeling, indicates an improvement of the quality of the nano-electroerosive impact process.

Keywords: nanotube, electric arc, model, instability, gap width, surface, nano fabrication, powder metallurgy 